\title{
BEHAVIOUR OF ISOTROPIC POINT IN $\mathrm{LiKSO}_{4}$ CRYSTALS
}

\author{
V.J. STADNyK, M.O. Romanyuk and V.F. Vachulovich \\ Physics Department, Lviv I. Franko University \\ Lomonosova 8a, Lviv, 290005, Ukraine
}

(Received January 7, 1992; revised version January 27, 1993)

The temperature-spectral dependencies of refractive indices and birefringence in wide temperature $(77-1000 \mathrm{~K})$ and spectral $(250-850 \mathrm{~nm}) \mathrm{re}$ gions in $\mathrm{LiKSO}_{4}$ crystals were measured. The isotropic point of $\mathrm{LiKSO}_{4}$ crystals was investigated. It was shown that annealing and X-ray irradiation display insignificantly the isotropic point to a visible region of spectrum.

PACS numbers: 77.80.-e

\section{Introduction}

The $\mathrm{LiKSO}_{4}$ crystals (LPS) undergo an unusual succession of phase transitions (PT): high-temperature hexagonal-ferroelastic (incommensurate)-room-temperature hexagonal-incommensurate-ferroelastic orthorhombic phases [1-5]. In spite of considerable interest in unusual physical properties of LPS crystals, their optical properties have not been practically studied, except from the observation of domain walls, Raman and Brillouin scattering and measurements of birefringence temperature dependencies for one wavelength [4-9].

For the first time the isotropic point has been observed in wide temperature $(185-430 \mathrm{~K})$ and spectral $(240-355 \mathrm{~nm})$ ranges by studying temperature-spectral dependencies of refractive indices and birefringence $\Delta n_{a}$ [10]. The purpose of the present paper is to investigate the influence of ageing, annealing and X-ray stimulated defects on the character and reproduction of temperature-spectral dependencies of the LPS crystals isotropic point.

\section{Experiment}

The temperature-spectral dependencies of refractive indices were measured by a photographic Obreimov's method. The reper values of refractive indices were obtained by immersion Obreimov's method in spectral region $460-670 \mathrm{~nm}$ with 
accuracy of $\pm 2 \times 10^{-4}$. The photographic Obreimov's method is based on the fact that a difference in optical path

$$
\left(n_{\mathrm{cr}}-n_{\mathrm{s}}\right) d=k \lambda
$$

(where $n_{\mathrm{cr}}-$ refractive index of crystal, $n_{\mathrm{s}}$ - refractive index of surroundings (in our case $n_{s}=1$ ), $\lambda$ - wavelength) appears when a parallel light beam passes along the boundary between crystalline plate and air.

Taking into account the condition of extremum, the refractive index will be as follows:

$$
n_{\mathrm{cr}}(\lambda, T)=1+\frac{k \lambda}{d(T)},
$$

where $k$ - interference minimum,order, $d(T)$ - thickness of crystal at given temperature and determined by thermal expansion.

An apparatus for measurements of the refractive indices was constructed using a spectrograph DFS-8 with linear dispersion of $6 \AA / \mathrm{mm}$. The accuracy of temperature changes of refractive indices was about $\delta n= \pm 2-5 \times 10^{-5}$.

The method of determination of birefringence also used a photographic method of recording the interference picture in focal plane of spectrograph DFS-8. The birefringence was calculated according to the formula

$$
\Delta n_{i}(\lambda, T)=\frac{k \lambda}{d(T)}
$$

The reper values of birefringence $\Delta n_{i}$ for each crystallographical direction were determined using the known reper values of refractive indices.

All optical measurements within both ferroelastic phases were performed on single domain areas.

\section{Results and discussion}

\subsection{Refractive indices}

It was determined that LPS crystals are uniaxial, negative, with optical axis along $c$-axis in hexagonal phase $\mathrm{I}(T>930 \mathrm{~K})$. The refractive indices change almost linearly with temperature $\left(\mathrm{d} n_{a} / \mathrm{d} T=8.4 \times 10^{-5} \mathrm{~K}^{-1}, \mathrm{~d} n_{c} / \mathrm{d} T=\right.$ $8.8 \times 10^{-5} \mathrm{~K}^{-1}$ ) - see Fig. 1. The transition into a ferroelastic phase II at $930 \mathrm{~K}$ is characterized by an insignificant jump like changes of $n_{i}: \delta n_{a}=1.4 \times 10^{-4}$, $\delta n_{b}=3.5 \times 10^{-4}, \delta n_{c}=2.2 \times 10^{-4}$, that testifies a first-order PT. In phase II LPS are optically biaxial, negative, with the direction of acute bisectrix of optical axes angle along $c$-axis. The first-order PT takes place at $710 \mathrm{~K}$ into a hexagonal room-temperature phase $\left(\delta n_{a}=7.2 \times 10^{-4}, \delta n_{b}=10.7 \times 10^{-4}, \delta n_{c}=5.3 \times 10^{-4}\right)$, where LPS are also uniaxial, with optical $c$-axis. The refractive indices increase almost linearly with the decrease in temperature $\left(\mathrm{d} n_{a} / \mathrm{d} T=1.6 \times 10^{-5} \mathrm{~K}^{-1}\right.$, $\left.\mathrm{d} n_{c} / \mathrm{d} T=2.2 \times 10^{-5} \mathrm{~K}^{-1}\right)$. At $255 \mathrm{~K}$ on $n_{a}(T)$ and $n_{c}(T)$ dependencies a weak break indicating second-order PT into incommensurate phase IV was observed. At $185 \mathrm{~K}$ the crystal undergoes first-order PT $\left(\delta n_{a}=4 \times 10^{-4}, \delta n_{b}=10 \times 10^{-4}\right.$, $\left.\delta n_{c}=29 \times 10^{-4}\right)$. The crossing of dispersion curves $n(\lambda)$ indicates the existence of birefringence inversion $\Delta n(\lambda)$. 


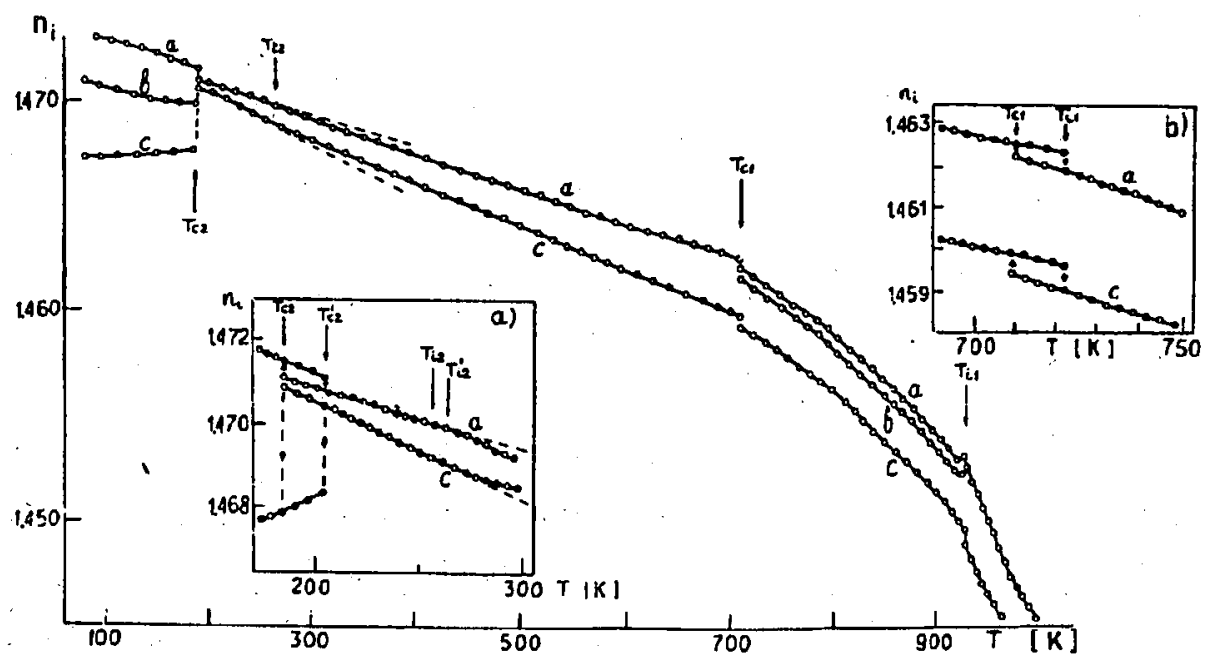

Fig. 1. The temperature dependencies of LPS crystals refractive indices for $\lambda=700 \mathrm{~nm}$ upon cooling. The insert: (a) the temperature dependencies of $n_{i}$ in PTs regions: phase III - phase IV - phase V ( 0 - cooling, $\bullet-$ heating); (b) the temperature dependencies of $n_{i}$ in PTs regions: phase II - phase III.

\subsection{Birefringence}

The temperature and spectral measurements of $\Delta n_{i}$ birefringence were carried out (Fig. 2) with the purpose of most careful study of the behaviour of an isotropic point in LPS crystals. The values of $\Delta n_{i}$ jumps during first-order P.Ts at $930 \mathrm{~K}\left(\delta \Delta n_{a}=13 \times 10^{-4}, \delta \Delta n_{b}=12 \times 10^{-4}, \delta \Delta n_{c}=25 \times 10^{-4}\right)$ and $710 \mathrm{~K}$ $\left(\delta \Delta n_{a}=14 \times 10^{-4}, \delta \Delta n_{b}=11 \times 10^{-4}, \delta \Delta n_{c}=26 \times 10^{-4}\right)$ were measured. At $287 \mathrm{~K}$ the equality of $\Delta n_{i}$ to zero was observed, which corresponds to an isotropic state of crystal. The birefringence value becomes negative and the change of crystal sign from negative to positive takes place $\left(n_{a, b}>n_{c} \rightarrow n_{a, b}<n_{c}\right)$. Further decrease in the temperature is accompanied by significant jumps of $\Delta n_{i}\left(\delta \Delta n_{a}=3.5 \times 10^{-3}\right.$, $\delta \Delta n_{b}=3.5 \times 10^{-3}, \delta \Delta n_{c}=3.5 \times 10^{-3}$ ). This agrees with the significant changes of the unit cell volume and indicates a first-order PT from phase IV into ferroelastic phase $\mathrm{V}$.

The gradual disappearance of interference minima with the decrease in temperature was observed, which corresponds to the change of an inclination sign of $\Delta n_{a}$ dispersion curves. The extremum point of $\Delta n_{i}$ dispersion corresponds to $\Delta n_{i} / \Delta T=0$. It was ascertained that the isotropic point moves to a long-wave range of spectrum. The presence of $\Delta n_{i}(\lambda, T)$ dispersion curve extremum gives a possibility to speak about the existence of a second point in IR region which moves to a visible region with increase in temperature (Fig. 3). The interference minima have completely disappeared in the region of an interference picture observation during first-order $\mathrm{PT}$ at $185 \mathrm{~K}$, which corresponds to the isospectral state of crystal (the birefringence becomes equal to zero in the whole spectral region $(250-850 \mathrm{~nm})$ under investigation). 


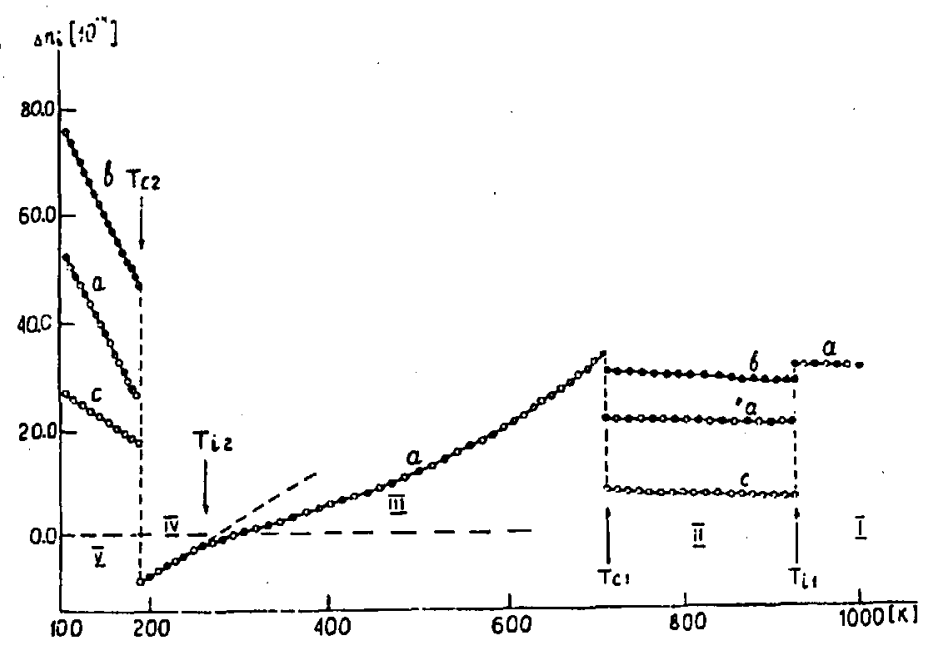

Fig. 2. The temperature dependencies of birefringence for the annealed LPS samples $(\lambda=500 \mathrm{~nm})$.

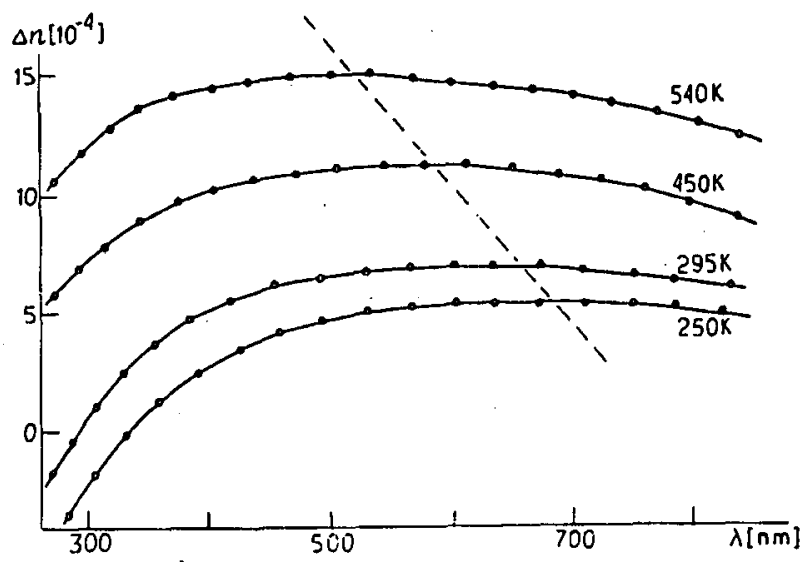

Fig. 3. The birefringence dispersion dependencies of annealed LPS samples.

\subsection{Isotropic point}

It was determined from the temperature dependencies of $n_{i}$ and $\Delta n_{i}$ that the isotropic point $\lambda_{0}$ of LPS crystals moves to a long-wave region of spectrum. The temperature motion of an isotropic point changed at $255 \mathrm{~K}$. The isotropic point disappeared at $185 \mathrm{~K}$. It appeared at $205 \mathrm{~K}$ upon heating and its temperature variation corresponds to the temperature variation upon cooling (Fig. 4, curve 1). The isotropic point was observed up to $240 \mathrm{~nm}$ at $430 \mathrm{~K}$. The above investigations were carried out on the samples annealed during 100 hours at 400,600 and $700 \mathrm{~K}$. 




Fig. 4. The spectral-temperature diagram of isotropic state of the optical indicatrix for annealed (curve 1), newly prepared (curve 2), aged (curve 3 ) and X-ray irradiated during two (curve 4) and four (curve 5) hours LPS crystals.

The displacement of $\lambda_{0}(T)$ curve to UV region by nearly $20 \AA$ for newly prepared samples was observed, but the character of the curve was reproduced almost completely. The discrepancy of PT point for newly prepared samples was observed: hexagonal modification III - incommensurate phase IV second-order PT took place at $260 \mathrm{~K}$, and first-order PT into ferroelastic phase IV at $180 \mathrm{~K}$. The hysteresis values were equal to $30 \mathrm{~K}$ (P'T at $210 \mathrm{~K}$ ) for first-order PT, $12 \mathrm{~K}$ (PT at $272 \mathrm{~K}$ ) for second-order PT under heating, $20 \mathrm{~K}$ (PT at $205 \mathrm{~K}$ ) and $10 \mathrm{~K}$ (PT at $265 \mathrm{~K}$ ) for the annealed samples.

The influence of X-ray irradiation on the behaviour of the isotropic point was studied. The isotropic point was displaced by nearly $30 \AA$ into visible region for two-hour irradiated samples and by $40 \AA$ for four-hour irradiated samples. Moreover, the displacement of PT point after X-ray irradiation was found out.

The study of aged crystals shows that the isotropic point was displaced by $50 \AA$ into visible region for 5 -year-old self-restrained crystals in comparison with newly prepared samples. Ageing did not influence the behaviour of the isotropic point for the annealed samples. For the 100-hour annealed samples concincidence of $\lambda_{0}(T)$ curves with accuracy $\pm 2 \AA$ was observed.

It is known that LPS phase IV is incommensurate, i.e. isotropic point passes through incommensurate phase which is characterized by the "phase solitons", fixed by crystal lattice defects. The "crystal water" type defects are predominant in crystals grown from aqueous solutions. Annealing of crystals leads to the decrease in hydroxyl group $\mathrm{OH}^{+}$density, i.e. to the decrease in "crystalline water" type defects. The change of the present defects density leads to the discrepancy of the 
properties measured in various regimes. Therefore, the discrepancy of $\lambda_{0}(T)$ curves for newly prepared, annealed and aged samples and the coincidence of these curves for annealed samples were not a surprise. The isotropic point behaviour after $\mathrm{X}$-ray irradiation was conditioned by the formation of radiation defects, whose concentration is proportional to the time of irradiation for small doses. The defects lead to a breach of the soliton regular distribution and to a change of the crystal isotropic state.

The isotropy condition can be obtained from Sellmeier's dispersion formula. For uniaxial crystals the refractive indices for ordinary and extraordinary beams are equal at a birefringence inversion point. The equality of expressions for $n_{\circ}$ and $n_{\mathrm{e}}$ refractive indices gives

or

$$
\frac{B_{\mathrm{e}} \lambda_{0 \mathrm{e}}^{2} \lambda^{2}}{\lambda^{2}-\lambda_{0 \mathrm{e}}^{2}}-B_{\mathrm{e}}^{\prime} \lambda^{2}=\frac{B_{0} \lambda_{0 \mathrm{o}}^{2} \lambda^{2}}{\lambda^{2}-\lambda_{0 \mathrm{o}}^{2}}-B_{\circ}^{\prime} \lambda^{2}
$$

$$
\lambda^{4}-b \lambda^{2}-c=0
$$

where

$$
b=\frac{B_{\mathrm{e}} \lambda_{0 \mathrm{e}}^{2}}{B_{\mathrm{e}}^{\prime}-B_{\mathrm{o}}^{\prime}}+\lambda_{0 \mathrm{e}}^{2}+\lambda_{0 \mathrm{o}}^{2}, \quad c=\lambda_{0 \mathrm{e}}^{2} \lambda_{0 \mathrm{o}}^{2}\left(\frac{B_{\mathrm{o}}-B_{\mathrm{e}}}{B_{\mathrm{e}}^{\prime}-B_{\mathrm{o}}^{\prime}}-1\right) .
$$

The solution of Eq. (5) determine the wavelength $\lambda_{0}$ - the point of birefringence inversion

$$
\lambda_{0}=\left\{\left[b \pm\left(b^{2}+4 c\right)^{1 / 2}\right] / 2\right\}^{1 / 2} .
$$

The expression for the displacement rate of isotropic point may be obtained from (7):

$$
\frac{\mathrm{d} \lambda_{0}}{\mathrm{~d} T}=\frac{2\left[b \pm\left(b^{2}+4 c\right)^{1 / 2}\right](\mathrm{d} b / \mathrm{d} T)(\mathrm{d} c / \mathrm{d} T)}{\left[b \pm\left(b^{2}+4 c\right)^{1 / 2}\right]^{1 / 2}\left(b^{2}+4 c\right)^{1 / 2}} .
$$

It was shown that for the LPS crystals in a room-temperature hexagonal phase: $\mathrm{d} \lambda_{0} / \mathrm{d} T=5 \AA / \mathrm{K}$ for annealed samples (curve 1, Fig. 4); $4.7 \AA / \mathrm{K}$ for newly prepared samples and $4.6 \AA / \mathrm{K}$ for $\mathrm{X}$-ray irradiated samples (curve 2 and 3 ). In the incommensurate phase IV: $\mathrm{d} \lambda_{0} / \mathrm{d} T=4.8 \AA / \mathrm{K}, 4.5 \AA / \mathrm{K}$ and $4.3 \AA / \mathrm{K}$, respectively.

The $\lambda_{0}(T)$ spectral-temperature dependence of LPS crystals is well described by the formula

$$
\lambda_{0}(T)=A \exp (b-\alpha T)+B,
$$

where $B=240 \mathrm{~nm}, A=1.15 \mathrm{~nm}, \alpha=0.017 \mathrm{~K}^{-1}, b=8.52$ for annealed samples, $B=241 \mathrm{~nm}, A=1.16 \mathrm{~nm}, \alpha=0.019 \mathrm{~K}^{-1}, b=8.50$ for newly prepared ones; $B=242 \mathrm{~nm}, A=1.18 \mathrm{~nm}, b=8.50, \alpha=0.016 \mathrm{~K}^{-1}$ for X-ray irradiated samples.

It was shown by the extrapolation of LPS crystals birefringence dispersion curves that a second isotropic point is $\approx 2010 \mathrm{~nm}$ (annealed samples), $\approx 2000 \mathrm{~nm}$ (newly prepared), $\approx 2040 \mathrm{~nm}$ and $\approx 2050 \mathrm{~nm}$ (X-ray irradiated samples). It moves to a visible region with temperature increase, which is analogous to the behaviour of the isotropic point of ammonium fluoroberylate (AFB) crystals [11-13].

In conclusion, the considerable anomalies of refractive indices and birefringence were observed in the LPS crystals. These anomalies are manifested as an 
unusual deformation of the optical indicatrix: uniaxial-biaxial-uniaxial-biaxial. It was shown that annealing and X-ray irradiation display insignificantly the isotropic point to a visible region of spectrum. Such behaviour is connected with stabilizing influence of annealing and X-ray irradiation on the space distribution as well as with change of the connection character of phase solitons and crystal lattice defects. It was shown that ageing of crystals influences its optical properties only for the newly prepared samples. The crystal annealing during 100 hours and more leads to the reproduction of $\Delta n_{i}(T), \lambda_{0}(T)$ and $n_{i}(T)$ dependencies.

The behaviour of the isotropic point in the region of second-order PT from phase III into phase IV and the hysteresis of $\lambda_{0}(T)$ change were studied.

\section{References}

[1] S. Fujimoto, N. Yasuda, H. Hibino, P.S. Narayan, J. Phys. D, Appl. Phys. 67, L35 (1984).

[2] D.P. Sharma, Pramana 13, 223 (1979).

[3] M.A. Pimenta, P. Echegut, Y. Luspin, G. Hauret, Phys. Rev. B 39, 3361 (1989).

[4] J. Mendes Filho, J.B. Moreira, F.E. Melo, F.A. German, Solid State Commun. 60, 1989 (1986).

[5] C.H.A. Fonseca, G.M. Ribeiro, R. Gazzinelly, A.S. Chaves, Solid State Commun. 46, 221 (1983).

[6] R. Cash, P.E. Tomashewski, J. Bornarel, J. Phys. C, Solid State Phys. 18, 915 (1985).

[7] P.E. Tomashewski, K. Lukaczewicz, Phase Transit. 4, 37 (1983).

[8] W. Kleemen, F.J. Schafer, Solid State Commun. 64, 1001 (1987).

[9] N.R. Ivanov, Ferroelectrics 64, 13 (1985).

[10] V.J. Stadnyk, V.M. Gaba, M.O. Romanyuk, Opt. Spektrosk. 68, 701 (1990).

[11] Z.M. Ursul, M.O. Romanyuk, V.M. Gaba, Opt. Spektrosk. 66, 665 (1988).

[12] M.O. Romanyuk, Z.M. Ursul, V.M. Gaba, A.P. Vaskiv, Opt. Anisotr. Sred 3, 105 (1987). 